\title{
Article \\ An In-Line Coaxial-to-Waveguide Transition for Q-Band Single-Feed-Per-Beam Antenna Systems
}

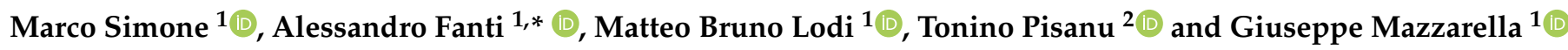 \\ 1 Department of Electrical and Electronic Engineering, University of Cagliari, 09123 Cagliari, Italy; \\ marco.simone@unica.it (M.S.); matteob.lodi@unica.it (M.B.L.); mazzarella@unica.it (G.M.) \\ 2 National Institute for Astrophysics, Cagliari Astronomical Observatory (INAF-OAC), \\ 09047 Selargius (CA), Italy; tpisanu@oa-cagliari.inaf.it \\ * Correspondence: alessandro.fanti@unica.it
}

Citation: Simone, M.; Fanti, A.; Lodi, M.B.; Pisanu, T.; Mazzarella, G. An In-Line Coaxial-to-Waveguide Transition for Q-Band Single-FeedPer-Beam Antenna Systems. Appl. Sci. 2021, 11, 2524. https://doi.org/ 10.3390/app11062524

Academic Editor: Pablo Padilla de la Torre

Received: 16 January 2021

Accepted: 24 February 2021

Published: 11 March 2021

Publisher's Note: MDPI stays neutral with regard to jurisdictional claims in published maps and institutional affiliations.

Copyright: (c) 2021 by the authors. Licensee MDPI, Basel, Switzerland. This article is an open access article distributed under the terms and conditions of the Creative Commons Attribution (CC BY) license (https:/ / creativecommons.org/licenses/by/ $4.0 /)$.

\begin{abstract}
An in-line transition between a coaxial cable and rectangular waveguide operating in Q-band (33-50 GHz) is presented. The aim of the work is to minimize the modifications in the waveguide to the strictly necessary to overcome the manufacturing issues due to the high frequencies involved. In addition, the transition is compact and it does not increase the space occupation on the transverse section, this suggests its application in horn antennas clusters arrangement. The operating principle consists of both a modal conversion and an impedance matching between the devices. The modal conversion is realized in an intermediate region, where the coaxial penetrates in the waveguide: the device geometry is designed so that the electric field in the transition is a trade-off between the TEM mode of the coaxial and the $\mathrm{TE}_{10}$ of the guide. A shaped waveguide backshort and a reactive air gap in the coaxial cable co-participate to achieve the matching. An optimized Chebyshev stepped transformer completes the transition to fulfil the impedance mismatch with the full waveguide. The design issues and technological aspects are considered. The influences of the feeding pin misalignment, the presence of groove is included in the analysis and these practical aspects are discussed and numerically validated via the scattering parameters analysis of the proposed design. The return loss is higher than $25 \mathrm{~dB}$ over the whole Q-band.
\end{abstract}

Keywords: transition; waveguide; coaxial; impedance matching; end-laucher; ridge

\section{Introduction}

High frequency systems require transmission devices with low losses and dispersion [1]. Moreover, the performance of microwave $(300 \mathrm{MHz}-30 \mathrm{GHz})$ and millimetre-wave (30-300 GHz) devices as antennas [2] or waveguides [3] strongly depends on geometrical parameters whose dimensions are smaller than the operative wavelength [4], so that as the variation scale of the electromagnetic waves involved decreases (e.g., at the frequency of $50 \mathrm{GHz}$, the free space wavelength is about $6 \mathrm{~mm}$ ), thus calling for a higher precision and tight mechanical tolerances in the fabrication, to avoid long and complicated postrealization tuning procedures [1]. A higher precision is required in their fabrication. In this framework, microwave engineers tend to prefer devices whose geometry allows an easier manufacturing process when the operating frequency rises [1]. These issues make horn antennas the preferred solution in high frequency transmission systems with respect to planar or wire antennas: horn antennas are generally all metallic and are characterized by a simple geometry, easy construction and excitation [5]. Such features make the horn one of the typical feeds at millimeter-wave (mm-wave) frequency range, as a stand-alone antenna, array element [6] or as feed for a reflector system [7]. At mm-wave, the channel is characterized by a high signal attenuation which limits the long distance transmission capability of a stand alone antenna and requires the applications of large reflector dishes to obtain an high directivity [8]. Since a high-directive radiation limits the beamwidth [9], the need to 
guarantee the communication over a wide angular range requires a multiple narrow-beam radiation, which translates in a single-feed-per-beam (SFB) antenna configuration [10].

Satellite communications exploit multiple-beam reflector antennas (or, in particular cases, phased arrays [11]) to achieve a continuous coverage over a desired field-of-view on terrestrial surface [10]. Since a different feed is required per each beam, the need to cover all the directions in a determinate angular range is satisfied with a dense cluster of horns [12,13]. A similar multiple horn-fed reflector antenna system is employed in radio-astronomy too [14], where the low power of the typical signals involved requires a large parabolic dish to collect the incoming radiation and focus it in a feed.The feed has a composite geometry, and a certain numbers of horn antennas are placed close each other in the focal point, providing a multi-beam radiation where the beams system covers a wide span of the sky [15]. Since not the whole radio frequency (RF) system in charge of the signal processing consists of waveguide (WG) components, a suitable transition is required to connect the horn to the RF front-end without degrading the signal. The traditional E-probe transition requires the orthogonal alignment of the second device with respect to the axis of the horn [16], this increases employment in the transversal area and limits the possibility of creating a cluster with a dense concentration of horn feeds, especially in the mm-wave range. In addition, the relative position between the reflector and the horn-feed is a design parameter which can be limited by the E-probe feed configuration. To overcome such limitations, the $\mathrm{TE}_{10}$ mode of the horn waveguide can be coupled to the field configuration in the downstream devices through an in-line transition which keeps the same direction of the field and does not increase the transversal size and does not concur to the blockage effect [10]. Microstrip and coaxial cable can be employed for such a purpose, and different solution have been studied, by shaping the end-launcher [17] and studying configurations which ease the connection between the devices [18], or involving gap-waveguides to improve the matching [19]. However, usually high-performance systems have coaxial connections [14]. For this reason, coaxial-to-waveguide in-line transition is a common solution to feed horn antennas employed in reflectors.

The in-line coaxial-to-waveguide transition has been proposed since the 1960s [20] and it has been employed to feed open-waveguide arrays [21]. The L-shaped coaxial configurations $[22,23]$ are a first common technique to short the launcher, but a folded conductor is a challenging task, especially in mm-wave technology, and it is overcome by patch antenna launchers [24] or by shorting the launcher directly on a tapered ridge or multi-section one quarter wavelength-length transformer ridges, which allow the matching over a wide bandwidth $[25,26]$. Despite the aforementioned complexity, the L-shaped solution has been widely used for horn antennas, even leading to patented devices, such as [27]. The bandwidth enlargement has been studied and it has been accomplished with different strategies, as a thicker pin (compared to the inner coaxial conductor) surrounded by a shaped cavity [28], or by widening both the width and the height of the waveguide [29]. Dielectric [30] or metallic [31] shaped fins have been shown to be an alternative to provide the desired matching. In [32], the matching is achieved over a wideband via an intermediate rectangular coaxial structure attached to a single ridge waveguide. These solutions presents relevant drawbacks, in particular the cumbersomeness of the technological strategy to meet the required transmission performances and modal conversion.

This paper proposes an in-line coaxial-to-waveguide transition with a compact geometry to obtain a good match over the whole Q-band (33-50 GHz). The aim is to reach the desired matching (typically, at least a return loss (RL) $>20 \mathrm{~dB}$ in radioastronomy) without increasing the transverse occupation and limiting the additional components to the strictly necessary, in order to obtain a satisfactory accuracy in the design despite the small operating wavelengths involved. This is an alternative solution to the previous work which involved a microstrip as end-launcher [33]. The shift between a microstrip and a coaxial end-launcher leads to several improvements. First of all, microstrip impedance and propagation constant are dispersive. To this aim, the design and the analysis are carried out by performing numerical experiments which take into account a number of 
potentially limiting technological scenarios. This dispersion is due both to a dispersivity of the dielectric constant and to the geometric dispersion of the microstrip [34]. The latter can be well modelled but only for "free space" microstrip, not in configurations as in [33]. On the other hand, the coaxial conductors have no geometrical dispersion, and the dielectric material dispersion is typically smaller than the dispersion of a microstrip slab [35]. As a consequence, the required bandwidth $(33-50 \mathrm{GHz})$ can be more easily achieved. Moreover, the unavoidable inaccuracy in the knowledge of the permittivity of a microstrip dieletric slab in the $\mathrm{Q}$ band reduces the overall accuracy of the matching and, as a consequence, of the performance [36]. This makes the coaxial cable the preferable choice in the radio astronomical receivers [14]. Finally, the field at the microstrip edges is rather large, and this increases the dielectric losses, which become the main loss source of the complete transition. The waveguide is a WR-22 $(\mathrm{a} \times \mathrm{b}=5.69 \times 2.845 \mathrm{~mm})$, whereas the coaxial cable is filled with Teflon $\left(\epsilon_{r}=2.1, \tan \delta=0.001\right.$, radius $\left.\mathrm{r}_{\mathrm{d}}=0.3386 \mathrm{~mm}\right)$ and ends in a pin with the same radius of the inner conductor $r_{i}=0.1136 \mathrm{~mm}$. The transition has to accomplish both a mode fitting and an impedance matching between the waveguide and launcher.

The in-line configuration keeps the same direction of propagation in both the components, so that the E-fields of the TEM mode in the coaxial cable and of the $\mathrm{TE}_{10}$ mode of the waveguide are parallel. In addition, the high wave impedance of the hollow waveguide must be matched to the $50 \Omega$ of the end launcher. Hence, the waveguide traversal geometry in proximity of the transition needs to be modified in order to both reduce its impedance and match its field distribution with the end launcher [28]. Then, the transition is matched to the full WR-22 through a stepped impedance transformer. The performance has been analyzed in terms of return and insertion loss.

\section{Design}

The high frequencies involved, up to $50 \mathrm{GHz}$, which correspond to a guided wavelength of $7.1 \mathrm{~mm}$ [37], strongly limit the degrees of freedom in the design. Since the precision required in microwave components is related to the operative wavelength, and usually it is smaller than an order of magnitude respect with the latter, transitions which match the coaxial cable using small metallic parts, such as folded pins [21,23], fins [30] or metallic bricks suspended with respect to the metallic walls as rectax [26] are rather demanding in realization accuracy when the operating frequency rises. Additionally, modification in the pin radius [28] or in its shape [32] is critical in Q-band due to the reduced physical dimensions. None of the mentioned solutions is proposed in the literature for operating frequencies up to $50 \mathrm{GHz}$. In the same time, it is important to reduce the number of design parameters, so it has been chosen not to modify the WG transverse section [29] and use a single ridge cascade.

The purpose of this work is to obtain a wideband transition without increasing the WG transverse occupation and with an as simple as possible geometry with respect to the solutions proposed in literature: no intermediate steps (except for a multistep impedance transformer) [29] or shaped pins [23,28] have been introduced in the design, and the waveguide transverse section has been modified only in the backshort, in the section where the launcher is inserted.

The geometry of the proposed transition is shown in Figure 1, where the left and the upper walls are removed for the sake of a clearer internal geometry display. The coaxial cable is inserted in the WG backshort of $(a \times b)$ size, where a circular hole with the same radius of the dielectric coaxial layer is realized, in central position with respect to the WG long side a. The pin is shorted to a ridge, this in turn is connected to a cascade of ridged waveguide steps which implement a Chebyshev stepped impedance transformer, in charge of achieving the impedance matching with the full WG [38]. 


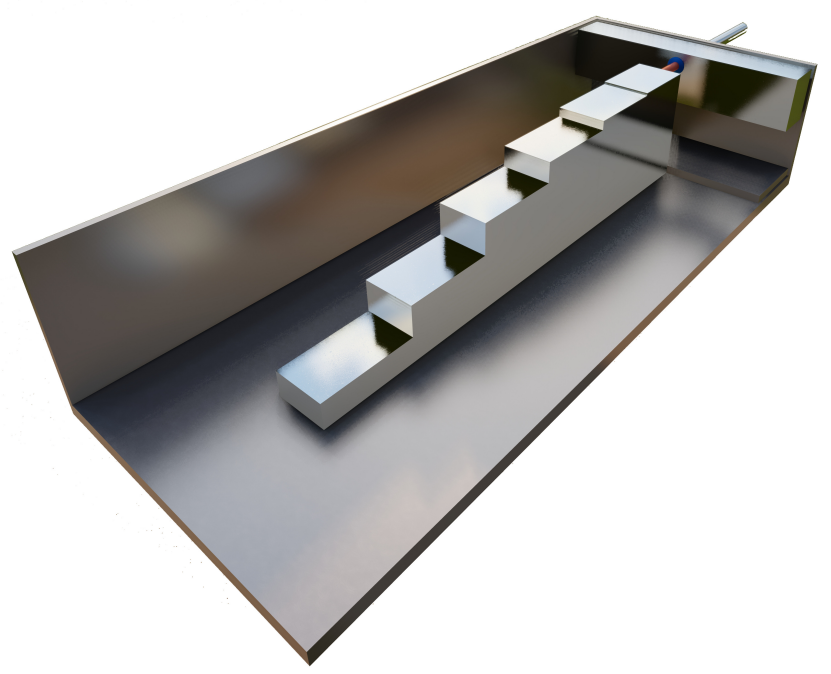

Figure 1. Geometry of the coaxial-to-waveguide transition: isometric view (the left and the upper walls are not displayed). The coaxial probe (displayed in orange) is inserted at the center of the waveguide (WG) backshort: the coaxial dielectric layer terminates in correspondence of the WG backshort face, whereas the inner conductor is shorted on the ridge. The resulting ridge WG structure is then matched to the hollow waveguide via a ridged steps cascade.

In order to achieve the modal conversion, only the coaxial inner conductor (pin) enters inside the guide, whereas the WG outer walls act as the coaxial outer conductor, so that the field configuration in this waveguide step is similar to a TEM. As a by product of this choice, no dielectric parts are needed in the mode converter as it would be required by using a microstrip end-launcher, and this significantly increases the achievable accuracy. In addition, such a field must be matched to a full WG. The introduction of a ridge for the pin short eases in the same time the modal conversion. As a matter of fact, by introducing an offset in the pin position along the short side b with respect to the center (Figure 2), and so approaching it to the WG upper wall, a large field is concentrated in the small gap $\mathrm{g}$ between the two aforementioned conductors. Such a field configuration is similar to a single-ridged waveguide (R-WG) whose ridge is centered in the long side with an height equal to the offset of the pin [33]. Moreover, the field lines in the pin section gather in the gap between the pin and the upper wall, and they are oriented parallel to the lines in the ridged area. Figure 3 shows the transverse field configuration in correspondence of the pin (a) and of the first ridge (b) in the waveguide: both cases concentrate the energy in the center of the wide side, whereas it is weak in the rest of the transverse section. In addition, the ridge reduces the WG impedance, making it comparable to the coaxial cable one [39]. 


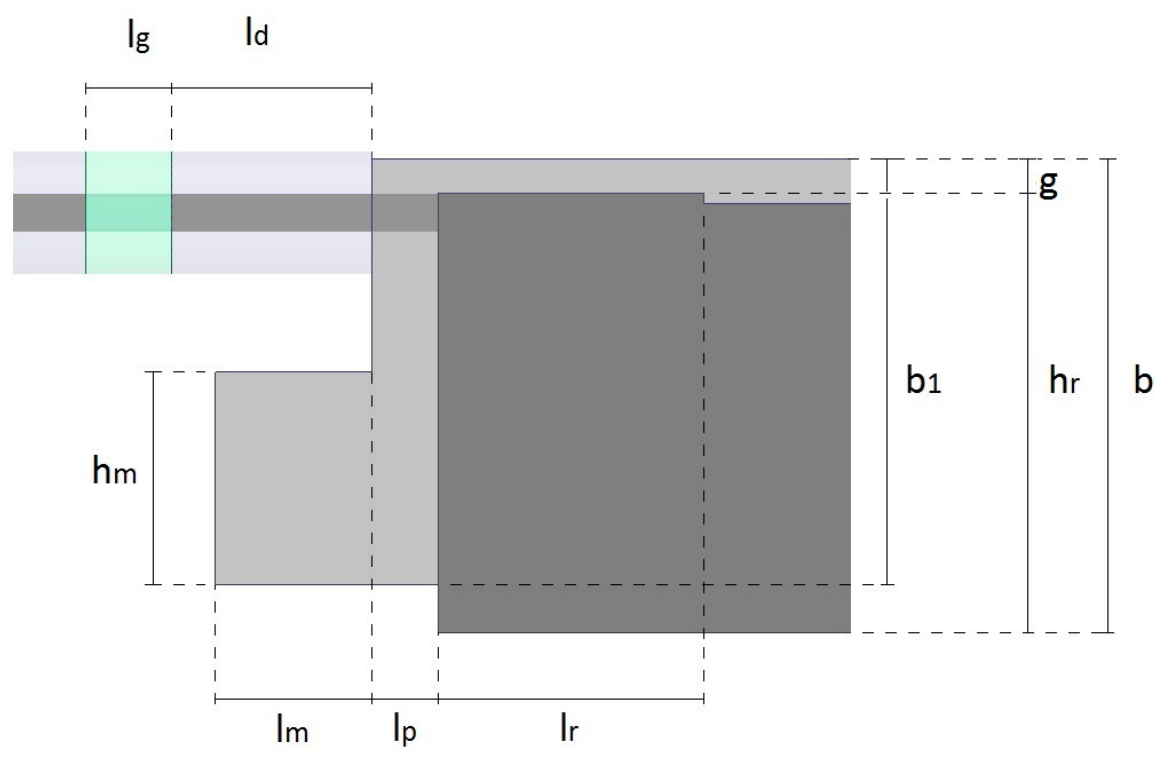

Figure 2. Geometry of the coaxial-to-waveguide transition: sectional lateral view. The pin $1_{p}$ long is shorted on a ridge $h_{r}$ high and $l_{r}$ long (in dark grey). The unridged WG step has a reduced height $b_{1}$, and a matching cavity of dimensions $\left(h_{m} \times l_{m}\right)$ is added along the backshort to improve the matching. The coaxial step highlighted in green indicates the $l_{g}$ long air gap inserted at a distance $l_{d}$ from the transition.

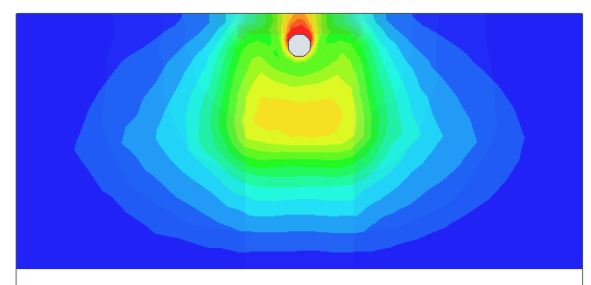

(a)

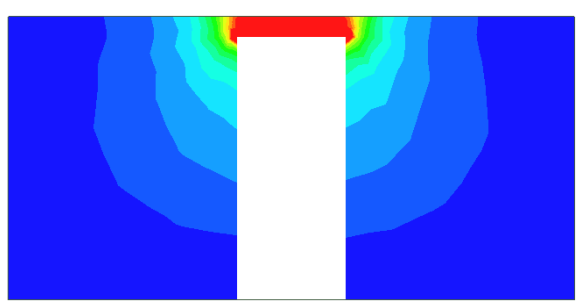

(b)

Figure 3. Field distribution in the mode transformation (in magnitude) at two different transverse sections. The white areas are metal filled. With reference to the Figure 2: (a) Electric field of the TEM mode on the cross-section at center of the $l_{p}$ long step where the coaxial pin penetrates in the WG; (b) Electric field of the $\mathrm{TE}_{10}$ mode on the cross-section at the center of $1_{\mathrm{r}}$ long ridged WG step.

Nevertheless, to improve the matching over a wide band, modifications in the backshort of the WG and in the coaxial are needed. Several strategies can be used to achieve this aim, as modifying the WG height $b$ in the $l_{p}$ step or the insertion of reactive components. In this work, as shown in Figure 2, the vertical side of the backshort is shortened from $b$ to $b_{1}$ in order to decrease the wave impedance to match it with the end-launcher and the ridged step, and a sort of rectangular cavity with the same width of the WG and respectively $l_{m}, h_{m}$ in depth and height is added. Such a cavity acts as a tuning stub whose reactive behaviour improves the impedance matching in the transition. Moreover, a reactance-series is introduced in the coaxial cable to further compensate the input reactance, realized through a discontinuity in the dielectric insulator. The Teflon is removed at $l_{d}$ distance from the transition to create a capacitance which balances the reactive parasitic effects inside the waveguide (highlighted in green in Figure 2). Such modifications do not increase the transverse section of the transition, maintaining the aim of a compact structure.

Finally, the ridged WG must be matched with the hollow WR-22 over the whole required bandwidth. This is realized through a multi-stepped Chebyshev impedance 
transformer based on ridges of progressive descending height. The bandwidth required for the impedance matching is connected with the number of steps in the transformer [40].

Because of the complexity of the geometry, there is not a precise guideline for the sizing of the whole transition, and an analytical model is not trivial to be defined due to the number of parameters involved. Early, the first ridge size is defined in order to suitably fit the coaxial impedance and the electric field distribution and in the same time not to complicate the manufacturing issue (e.g., a too short ridge can make the pin shorting challenging). After this, the backshort and the air gap in the end-launcher are sized together to obtain the desired matching: the first reduce the impedance in the first WG step, where the presence of the coaxial pin avoids the adoption of a ridge, whereas the gap balances the reactive component of the WG input impedance. The $l_{p}$ long section is the main part where the modal conversion applies [28]: it consists on a TEM structure with a rectangular outer conductor and a circular inner conductor, the coaxial pin, where the impedance is reduced by resizing the WG height $b_{1}$, and shaping the backshort with the cavity, rather than shaping and acting on the cylindrical pin radius, which is a more complicated technological solution, as done in [28]. In the same time, as aforementioned, the field in such a modal conversion step is concentrated the gap between the coaxial pin and the upper wall, by simulating the modal distribution in the downstream R-WG. With reference to Figure 2, the pin is $l_{p}=0.4 \mathrm{~mm}$ long and $g=0.205 \mathrm{~mm}$ distant from the upper wall of the WG. The ridge where the pin is shorted keeps the same hollow space above, so it results $h_{r}=2.64 \mathrm{~mm}$ high, $1_{\mathrm{r}}=1.2 \mathrm{~mm}$ long and $\mathrm{w}=1.1 \mathrm{~mm}$ wide. The hollow step inside the coaxial cable is $l_{\mathrm{g}}=0.52 \mathrm{~mm}$ long and it is placed $l_{\mathrm{d}}=1.2 \mathrm{~mm}$ distant from the pin. The Smith Chart in Figure 4 shows the effect of the air gap insertion. The backshort height is reduced to $b_{1}=2.56 \mathrm{~mm}$ in the step where the pin enters inside the $W G$, and a cavity $h_{m}=1.28 \mathrm{~mm}$ high and $l_{\mathrm{m}}=0.94 \mathrm{~mm}$ deep is added behind it.
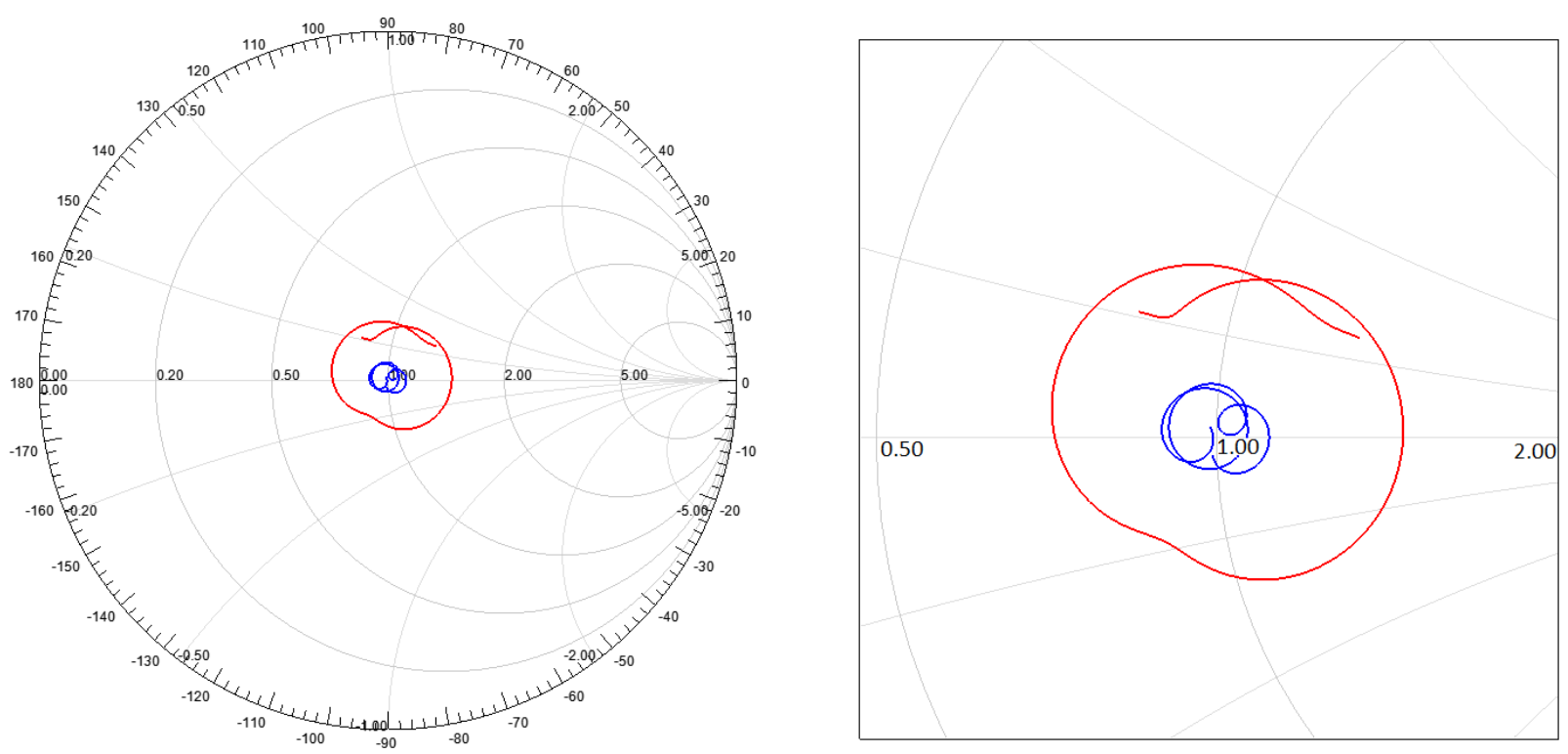

Figure 4. Effect of the air gap in the coaxial cable: the return loss with the air gap (blue line) and without (red).

The Chebyshev impedance transformer has been realized in 5 steps, which have proven to be the best trade-off between the RL performance and the specification of small transition. All the ridges have the same width of the first $(1.1 \mathrm{~mm})$. The requirement of high RL at such frequencies requires very precise size for the transformer steps, more than the values that can be obtained by the approximated formula for R-WGs [39]. Moreover, also the effects of the reactive phenomena due to the discontinuities between each couple of ridges must be traded by correcting the ridge lengths. To overcome these two issues, the Chebyshev impedance transformer has been optimized through the PSO algorithm, widely 
used in electromagnetic problems [41,42], in order to determine the lengths and the heights of the ridges which minimize the RL seen at the highest ridge section. The optimization procedure is based on the combined operation of MATLAB 2015 and a Finite Element Method (FEM) 3D electromagnetic solver (Ansys Electronics Desktop 2016.2), respectively in charge of the optimization algorithm script definition and of the electromagnetic performance evaluation, in order to minimize the overall RL in the required frequency band. A conceptual scheme of this process is explained in Figure 5. The algorithm considers as swarm positions the deviation of the R-WG geometrical parameters (in this case, the ridge heights and lengths) from the theoretical values calculated via the formula available in [39], then calculates the objective functions (which accounts for a maximum RL over the whole Q-band) through the FEM solver and iterates till convergence is reached. The working principle of such an optimization process has been explained in details in [33] and the optimized values of the ridges' size (displayed in Figure 6) are reported (in millimeters) in the Table 1.

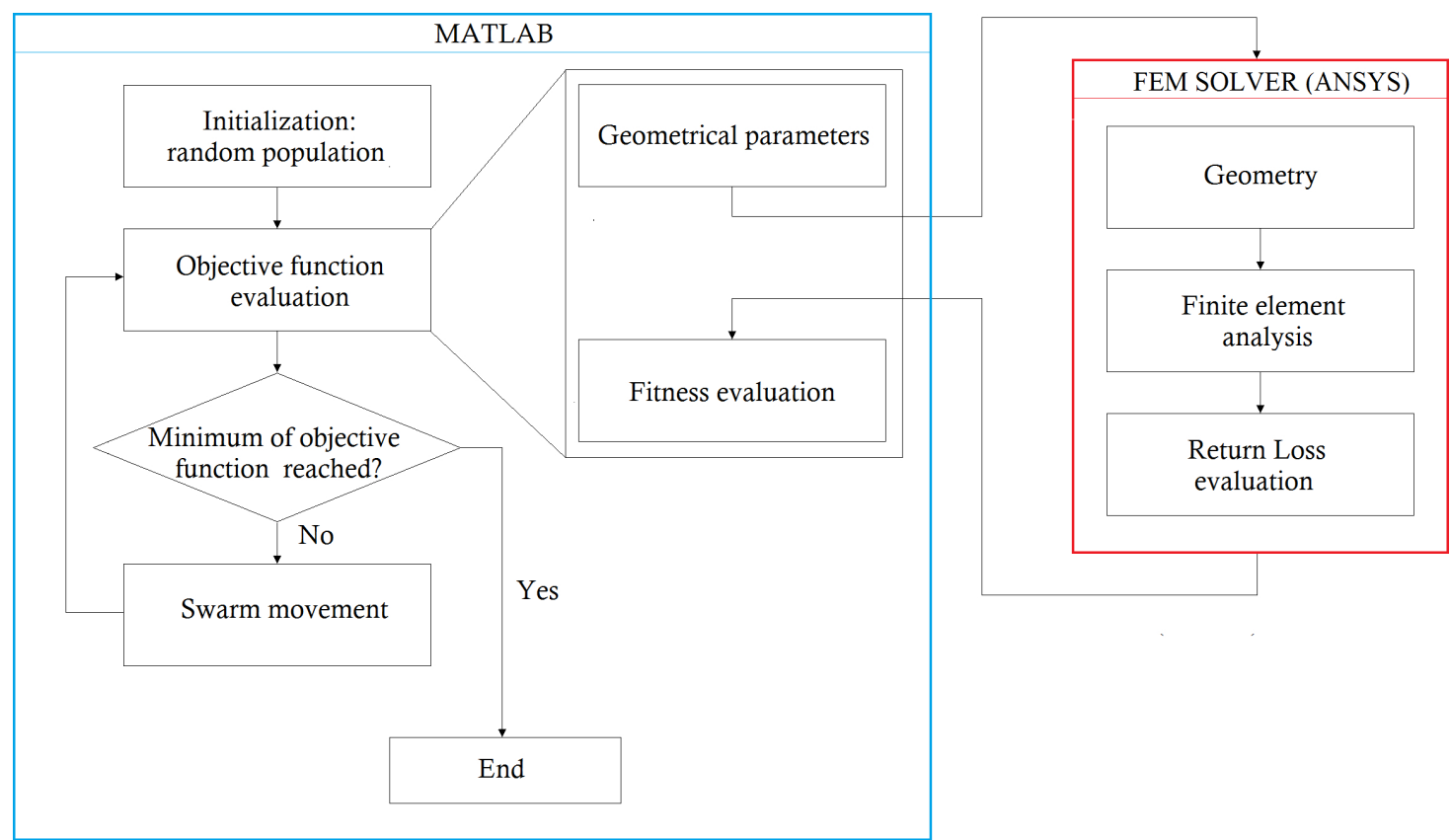

Figure 5. Summary of the PSO-based optimization algorithm for the Chebyshev transformer [33].

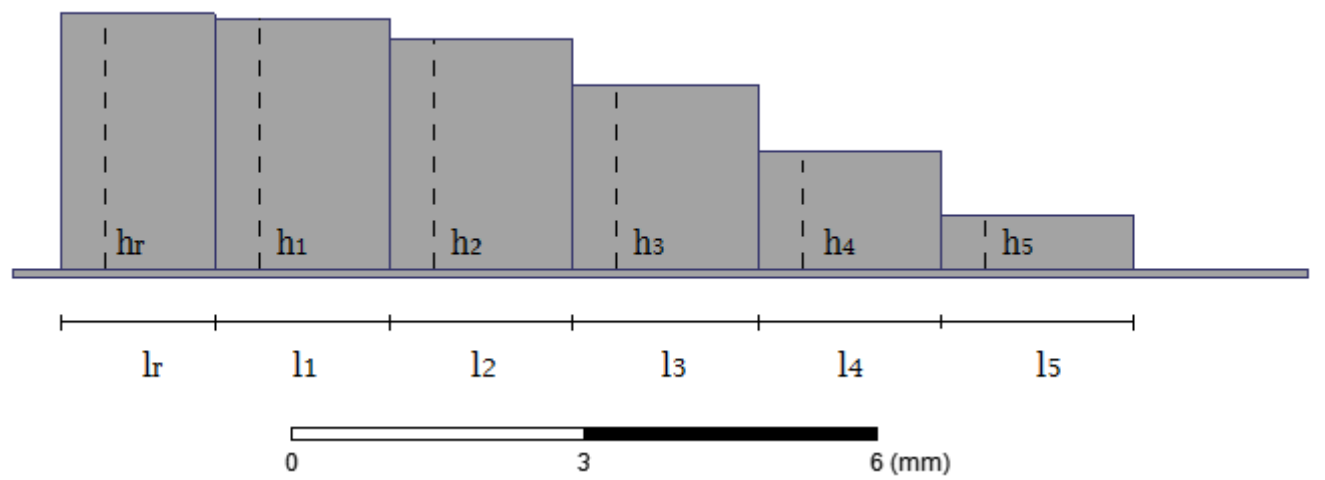

Figure 6. Ridge cascade structure: side view. The first ridge (of size $h_{r}, l_{r}$ ) is the same as in Figure 2, where the coaxial pin is shorted. The remaining ridges make up the Chebyshev impedance transformer, whose size $h_{i}, l_{i}, i=1, \ldots, 5$ are optimized via the PSO algorithm. 
Table 1. Geometrical size of the Chebyshev impedance transformer.

\begin{tabular}{ccc}
\hline Step & $\mathbf{h}[\mathbf{m m}]$ & $\mathbf{1}[\mathbf{m m}]$ \\
\hline 1 & 2.58 & 1.81 \\
2 & 2.37 & 1.9 \\
3 & 1.91 & 1.92 \\
4 & 1.22 & 1.9 \\
5 & 0.55 & 1.99 \\
\hline
\end{tabular}

\section{Results}

The transition was designed through a FEM electromagnetic solver, Ansys Electronics Desktop 2016.2. The waveguide and the coaxial outer conductor were modelled in aluminium, the coaxial inner conductor was modelled in copper. By considering from the air gap in the coaxial to the smallest ridge in the WR-22, the transition was $13.24 \mathrm{~mm}$ long. It is worth noting that the main part of the transition in terms of space occupation $(9.52 \mathrm{~mm})$ consisted of the Chebyshev impedance transformer, the rest of the matching network consisted only of the pin and the first ridge where this was shorted and it was $1.6 \mathrm{~mm}$ long inside the waveguide, $3.32 \mathrm{~mm}$ considering from the air gap in the coaxial cable to the same ridge (the backshort cavity lays inside this length), which was $0.55 \lambda_{0}$ at the higher frequency considered $(50 \mathrm{GHz})$.

\subsection{Performance}

The performance was evaluated in terms of scattering parameters between the transverse section of the coaxial cable upstream the air gap and the transverse section of the hollow waveguide, far enough from the Chebyshev transformer to avoid issue due to higher modes in the ridges' edges.

Figure 7 shows the return loss and the insertion loss. The RL was higher than $25 \mathrm{~dB}$ over the whole Q-band (BW\% > 40\%), guaranteeing a very good matching between the horn and the coaxial. However, the most remarkable result was the IL lower than $0.06 \mathrm{~dB}$ over the whole Q-band. This was significantly smaller compared with the microstrip-fed transitions, where values around $0.25 \mathrm{~dB}$ were typical [33], because the latter had a larger fraction of (lossy) dielectric, and, moreover, the electric field in the dielectric was far larger than the field of a coaxial cable. These results were also validated by using CST Microwave Studio, and it resulted in a deviation less than $1 \mathrm{~dB}$ in the whole frequency band (this evaluation is reported in Appendix A).

\subsection{Tolerance Analysis}

Since, as aforementioned in the introduction, the required accuracy and tight mechanical tolerances is one of the main issues at such frequencies [1], it is relevant to investigate some of the most critical parts in the design in order to estimate the tolerances in the manufacturing process. By performing accurate numerical experiments, as usually done when designing this type of RF devices, we expect to minimize post-realization tuning procedures and technological contingencies, while gaining relevant insights into the performance of the proposed in-line coxial-to-waveguide transition at Q-band.

The main issue is surely the connection between the pin and the waveguide. More precisely, the vertical offset with respect to the WG center is the most sensitive parameter: the gap g determines the characteristic impedance of the R-WG step immediately downstream the end launcher, so that it is matched to the latter, and in the same time it sets the impedance to be matched to the full waveguide through the Chebyshev impedance transformer. Therefore, we have evaluated the transition RL response over an interval of values of $g$ around the optimal value.

As shown in Figure 8, the RL was better than $20 \mathrm{~dB}$ over the whole $\mathrm{Q}$ band for a values range of $g$ equal to $0.195-0.215 \mathrm{~mm}$ (about $10 \%$ around the design value). In this range the ridge kept higher than the first Chebyshev transformer ridge $\left(\mathrm{h}_{\mathrm{c} 1}\right.$ high), further increase 
in $g$ degraded the performance due to the impedance mismatch between the two ridge heights $h_{r}, h_{c 1}$. As a matter of fact, by considering only the transition stadiums between the air gap and the first ridge as in Figure 9 (in the following, the WG step with the $h_{r}$ high ridge was named $W_{\mathrm{r}}$ ), the matching showed a higher tolerance, as displayed in Figure 10. As reported in the graph, the RL was evaluated by varying $g$ in the range $0.18-0.22 \mathrm{~mm}$, which was a variation of $20 \%$ around $0.2 \mathrm{~mm}$. The RL kept higher than $20 \mathrm{~dB}$ in all the variation range, by assuring a good tolerance for such a parameter and a suitable matching between the end launcher and the R-WG. It follows that, in the previous test, a significant contribution to the performance degradation was due to the different impedance value in the $\mathrm{WG}_{r}$ with respect to the theoretical value the Chebyshev transformer was matched to.

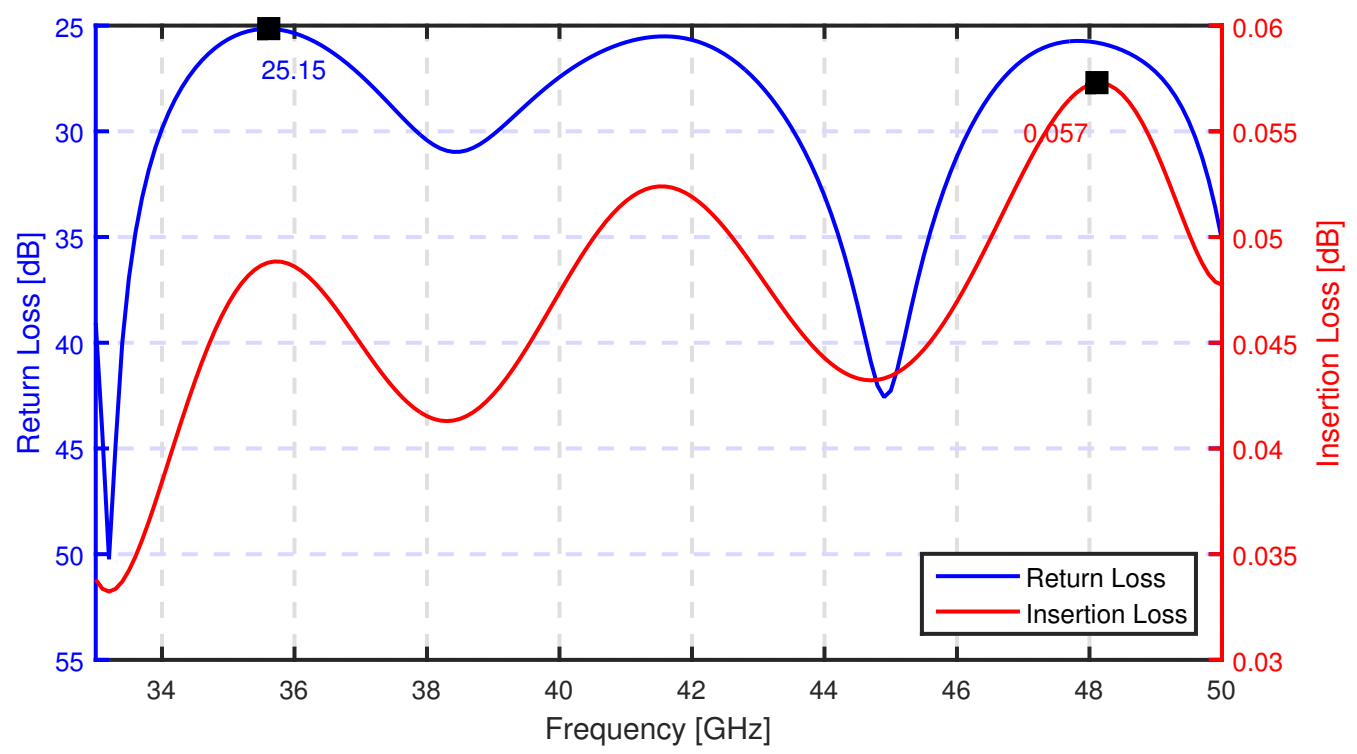

Figure 7. Return loss and insertion loss evaluated in the section between the transverse section of the coaxial cable upstream the air gap and the transverse section of the hollow waveguide. This section is far enough from the Chebyshev transformer to avoid issues due to higher modes in the ridges' edges.

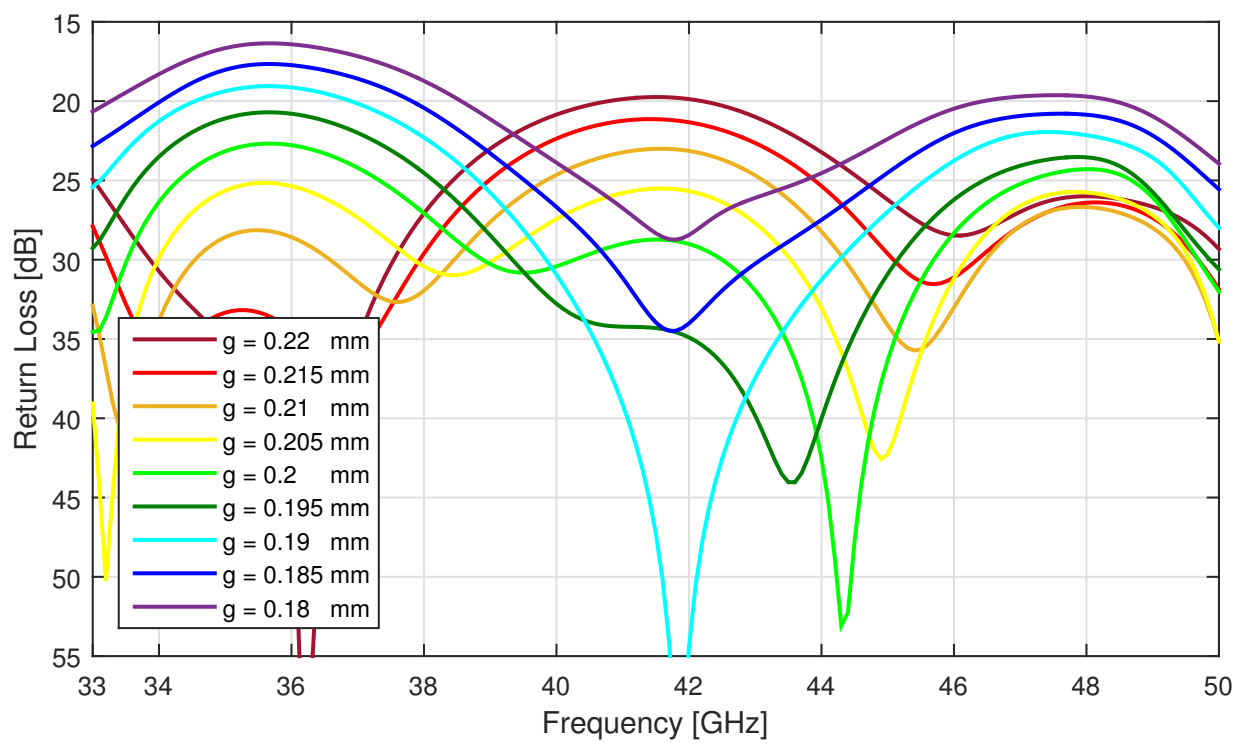

Figure 8. Tolerance analysis for the vertical offset of the pin insertion. The return loss is evaluated by varying the gap $g$ between the pin and the upper WG wall. 


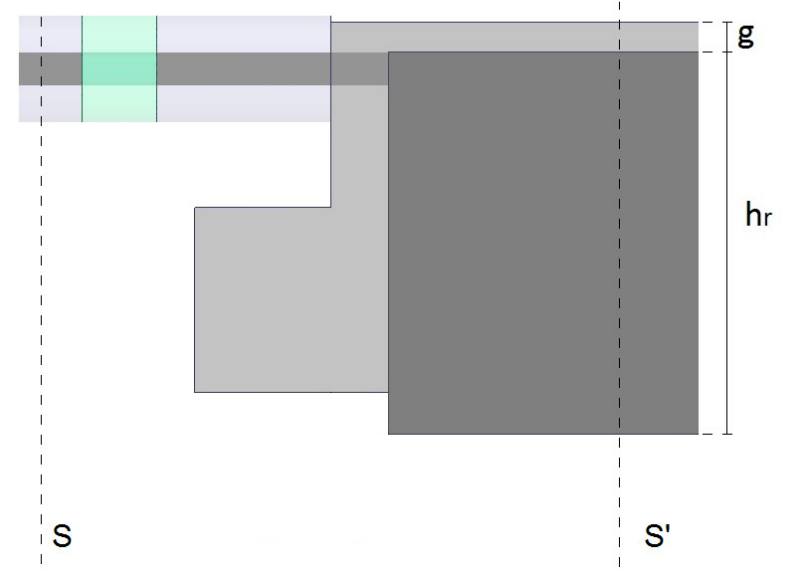

Figure 9. Section of the device involved in the second gap test: it is evaluated the performance by considering the device step from $S$ section (upstream the air gap) to $S^{\prime}$ (in the $W_{G_{r}}$ step) and by varying the $g$ parameter, which is related to the position of the pin insertion and to first ridge height. The RL is evaluated in input to the S section.

Therefore, in order to reduce the performance degradation of the manufactured device, it is worthwhile to split the design and manufacturing process in two steps (feasible, for instance, with milling or 3D printing technologies [43]). The first consists in the realization of the block from the coaxial end launcher to the aforementioned $\mathrm{WG}_{r}$ step, once the real $\mathrm{h}_{\mathrm{r}}^{*}$ value is measured (and, the input impedance in $\mathrm{WG}_{r}$ towards the launcher is known), the ridge-stepped Chebyshev transformer is designed and optimized with respect to $h_{r}^{*}$. In this way, a partial compensation of the inaccuracies given by unavoidable errors in the the construction process is obtained.

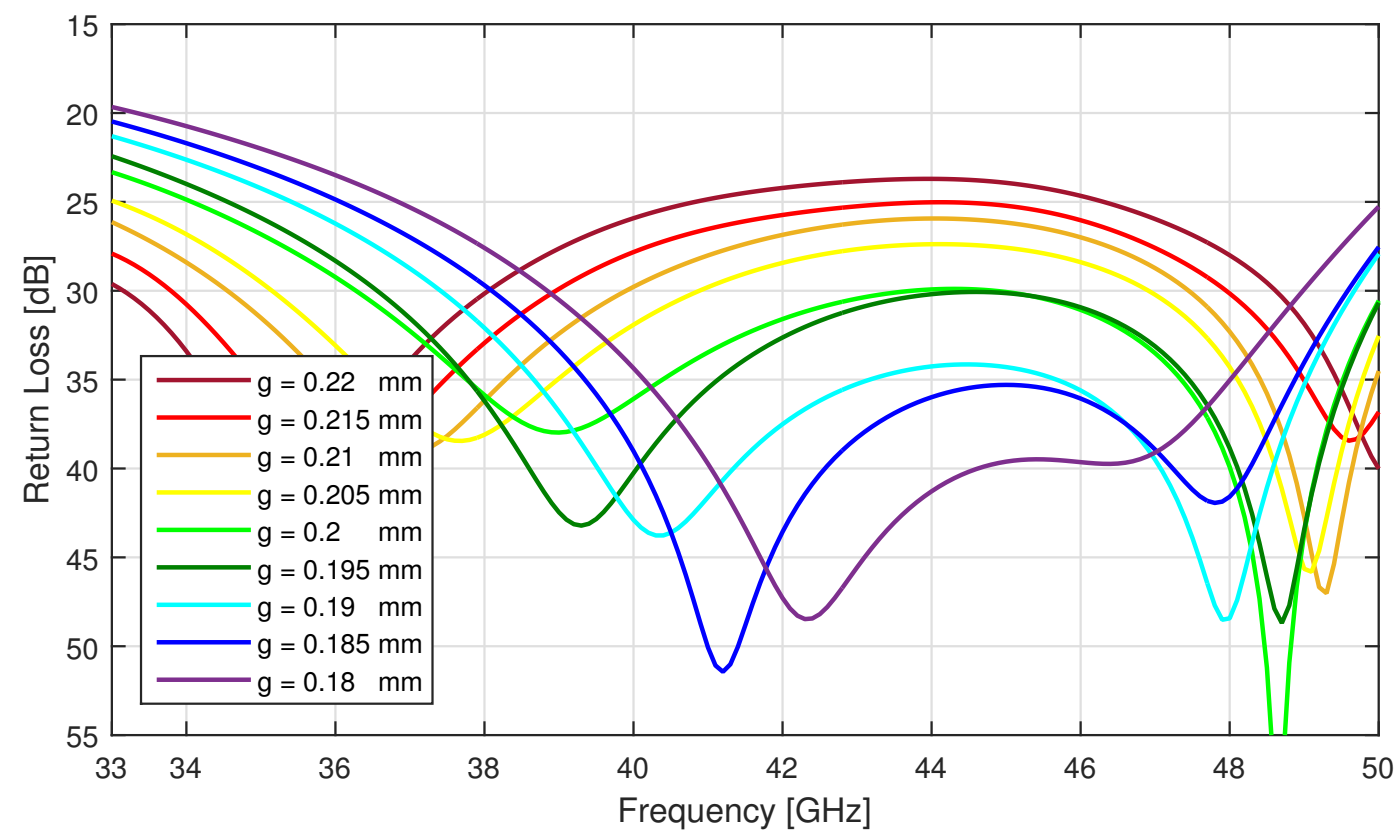

Figure 10. Return loss of the transition step shown in Figure 9. The g parameter modifies the impedance in the $W_{\mathrm{r}}$ step and introduces a mismatch with the coaxial cable which affects the RL.

Another significant matter is the short between the coaxial pin and the ridge. The proposed solution, shown in Figure 11, is to create a groove in the ridge, with a width equal to the pin diameter, and to short-circuit via the application of a two-component glue (like 
a silver-filled epoxy paste, as EPO-TEK H20E). The significant size of the groove was small compared to the operative wavelength, so it would not affect the performance in a relevant way and the ridged step impedance kepteps almost the same. Figure 12 shows the deviation in the return loss for a $1 \mathrm{~mm}$ long groove compared with the base case, and confirmed a negligible deviation in the performance. However, our refined analysis considered several possible scenarios where the pin position was not perfectly centred along the WG but it presented an offset in the H-plane. A wider groove was considered, modelled as EPO-TEK H20E filled, and several potential errors due to the non perfect alignment of the end-launcher were considered (the cases are shown in a wide panel displayed in Figure 13):

1. the original RL presented in Figure 7 (reference case);

2. a simple lateral shift of $0.4 \mathrm{~mm}$ (which covered a $0.8 \mathrm{~mm}$ range over a $1.1 \mathrm{~mm}$ ridge width) of the groove along the ridge width (Figure 13a,b);

3. a groove (wide twice the pin diameter, equal to the $40 \%$ off the ridge width) whose glue thickness was $20 \mu \mathrm{m}$ higher than the metallic ridge, with a lateral shift of $0.1 \mathrm{~mm}$ of the pin (the maximum available in the groove) (Figure 13c,d);

4. a groove (wide twice the pin diameter, equal to the $40 \%$ off the ridge width) whose glue thickness was $20 \mu \mathrm{m}$ higher than the metallic ridge and the direct contact was missing between the pin and the ridge, with a lateral shift of $0.1 \mathrm{~mm}$ of the pin (the maximum available in the groove) (Figure 13e,f).

The graph in the Figure 14 reports the RLs over this study: all the cases considered keep the RL higher than $20 \mathrm{~dB}$ over the whole $\mathrm{Q}$ band, this assured a good tolerance on the pin shorting to the ridge. It is worth noting, by considering cases 3 and 4 , that a non-direct contact of the pin was well compensated by the silver-filled glue, since the RL retained a value higher than $20 \mathrm{~dB}$, thus implying that, in this unfortunate manufacturing error, the glue acted as a conductive pathway from the pin, still ensuring a good signal integrity. Further analysis showed that a groove width three times the pin diameters (with the relative pin misalignment) kept the RL over $18 \mathrm{~dB}$ over the whole Q-band. In Figure 15 is shown the field distribution on the cross-section at center of the $l_{p}$ long step where the coaxial pin penetrated in the WG: the misalignment of the pin and the grooved shorting did not affect the $\mathrm{TE}_{10}$ mode in the WG. Therefore, from our simulations, a relevant insight and useful feedback to the future manufacturing step was found.

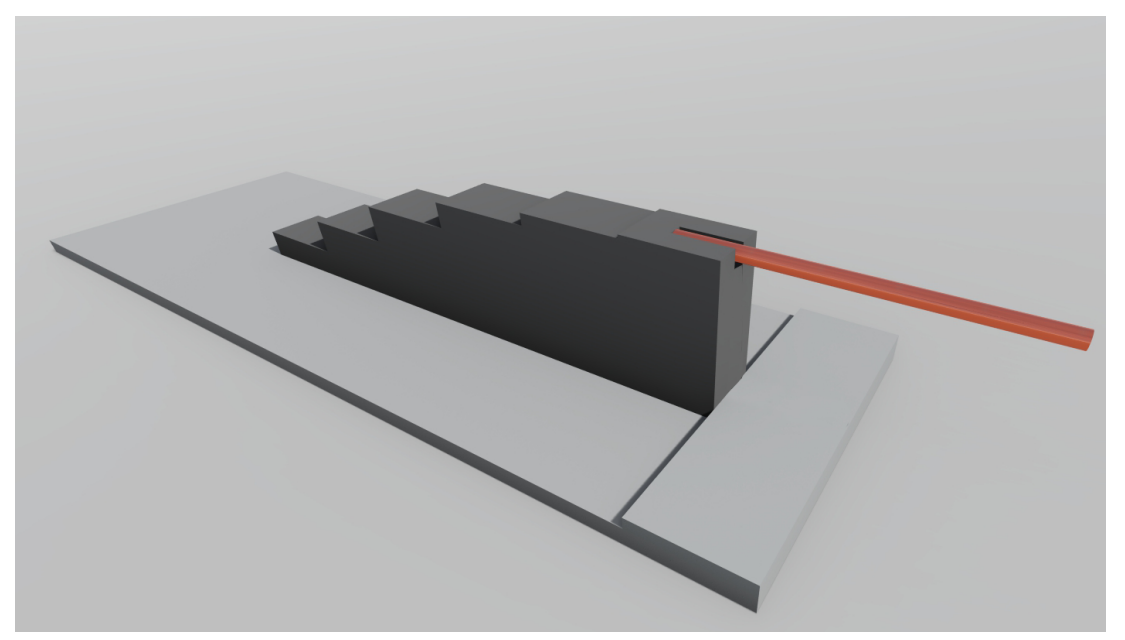

Figure 11. Practical shorting of the pin on the ridge. The coaxial pin (in orange) is inserted in a groove realized in the first ridge (gray), and pasted via a conductive glue. 


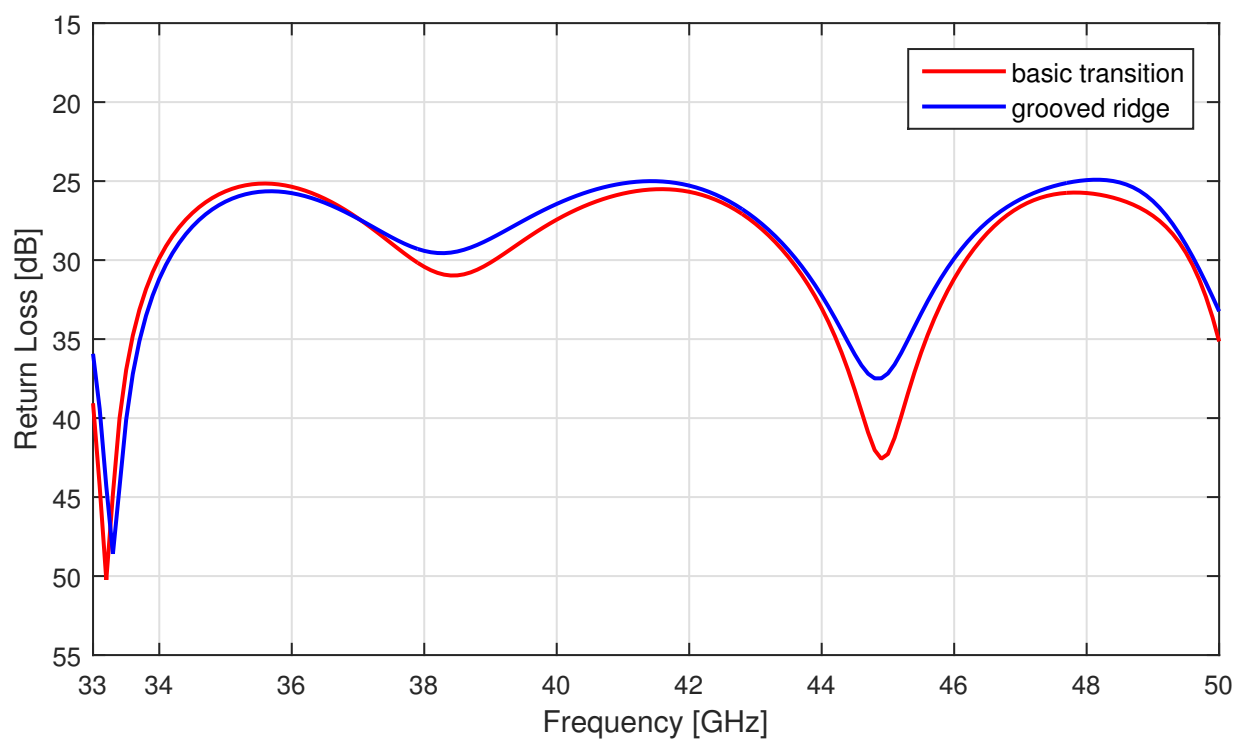

Figure 12. Return loss comparison: the grooved short displayed in Figure 11 (blue line) does not introduce a relevant variation with respect to the return loss of the initial design shown in Figure 7.
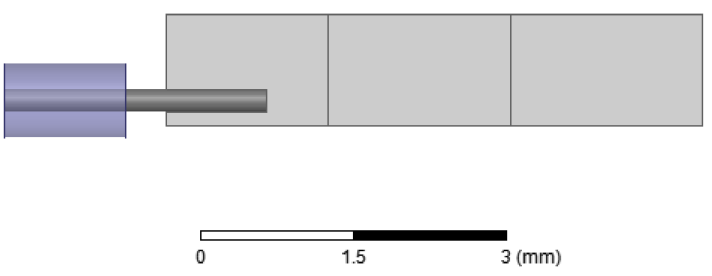

(a) case 2: top view
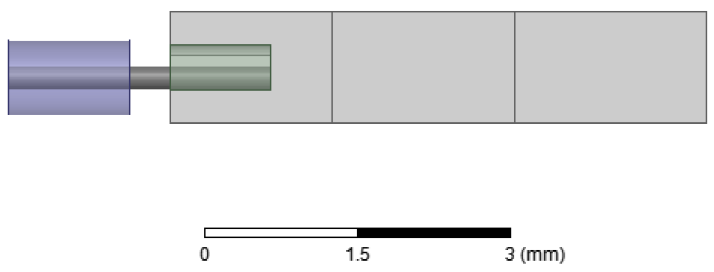

(c) case 3: top view
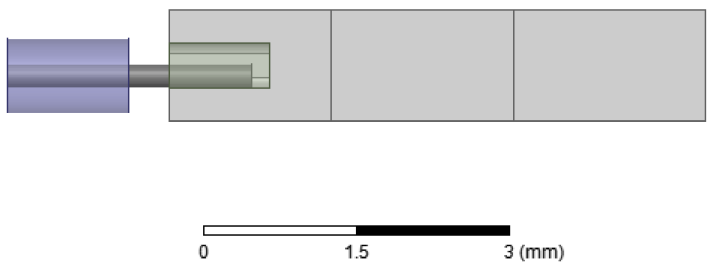

(e) case 4: top view

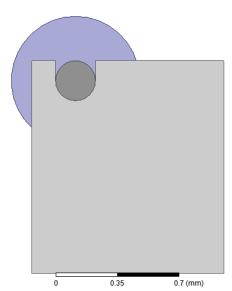

(b) case 2: front view

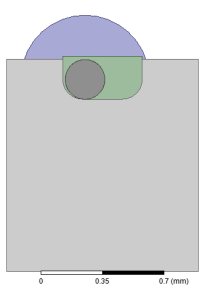

(d) case 3: front view

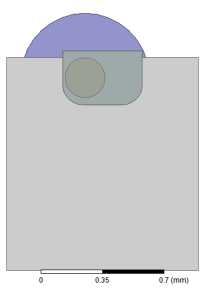

(f) case 4: front view

Figure 13. Geometry of the cases tested for the non perfect alignment of the end-launcher (gray) on the ridge (light gray), displayed in top and frontal view: $(\mathbf{a}, \mathbf{b})$ case $2,(\mathbf{c}, \mathbf{d})$ case $3,(\mathbf{e}, \mathbf{f})$ case 4 . The glue which fills the groove is displayed in green. 


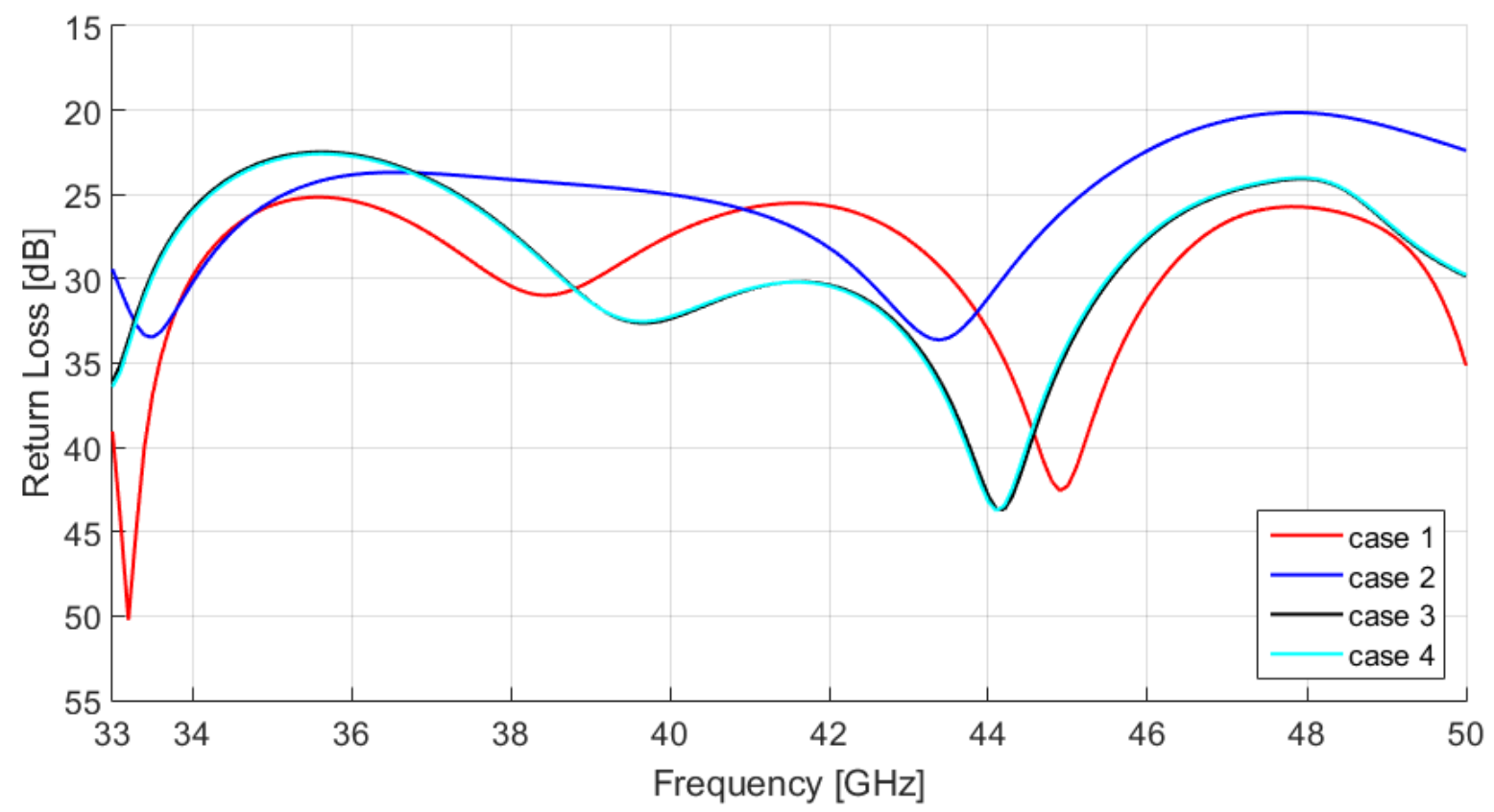

Figure 14. Return loss comparison between the tests on the groove and pin incorrect centering.
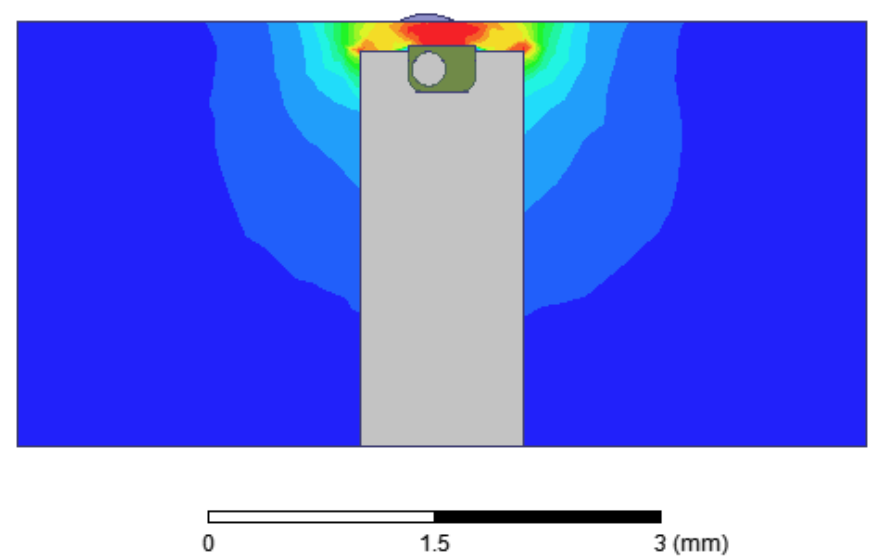

Figure 15. Field distribution in case 4 , on the cross-section where the pinis shorted to the ridge. The ridge and the pin are displayed in gray, and the EPO-TEK H20E in green. The pin misalignment and its non-direct contact with the ridge does not affect the $\mathrm{TE}_{10}$ mode.

\subsection{Discussion}

The transition performance was evaluated in terms of RL and IL over the whole Qband, and confirmed the suitability of such an in-line configuration. In the Table 2, a brief comparison of the similar works which constitute the state of the art is provided, taking into account both the in-line coaxial-to-waveguide (coax $2 \mathrm{wg}$ ) and the in-line microstripto-waveguide (us $2 \mathrm{wg}$ ) transitions and comparing for all the cases the bandwidth with $\mathrm{RL}$ higher than $20 \mathrm{~dB}$ : the proposed transitions with a comparable performance are all proposed at lower frequency ranges and adopt solutions which are too critical in Q-band, as a shaped pin [26,28] or a tunable adjustable fin [25]. As explained in the first paragraph of Section 2, these techniques introduce additional elements in the geometry, which translate in additional potential errors. 
Table 2. A comparative table on performance of the different transitions proposed in literature.

\begin{tabular}{cccc}
\hline Work & Structure & Simulated RL & BW\% \\
\hline$[17]$ & us2wg & $20 \mathrm{~dB}-\mathrm{BW}: 92-100 \mathrm{GHz}$ & 8.33 \\
{$[18]$} & us2wg & $20 \mathrm{~dB}-\mathrm{BW}: 8.35-9.6 \mathrm{GHz}$ & 7.18 \\
{$[19]$} & us2wg (gap WG) & $20 \mathrm{~dB}-\mathrm{BW}: 77-108 \mathrm{GHz}$ & 33.5 \\
{$[24]$} & coax2wg (patch launcher) & $20 \mathrm{~dB}-\mathrm{BW}$ 10-10.4 GHz & 3.9 \\
{$[25]$} & coax2wg with tunable fin & $20 \mathrm{~dB}-\mathrm{BW}: 2.5-4 \mathrm{GHz}$ & 46 \\
{$[26]$} & coax2wg with rectax & $20 \mathrm{~dB}-\mathrm{BW}: 3-4.8 \mathrm{GHz}$ & 46.15 \\
{$[28]$} & coax2wg with shaped pin & $20 \mathrm{~dB}-\mathrm{BW} 7.5-13 \mathrm{GHz}$ & 54 \\
\hline
\end{tabular}

The shorting between the coaxial pin and the ridge has been topic of several tolerance analysis to evaluate the design robustness respect to this manufacturing issue. The pin vertical offset has been analysed and shows an acceptable tolerance on the gap value, around the $20 \%$ (Figure 10). Moreover, the proposed solution to short the end-launcher to the waveguide does not affect the performance (Figure 12), and even if the pin is not perfectly centred in the ridge the RL keeps high enough (Figure 14) and the transverse field distribution keeps the $\mathrm{TE}_{10}$ mode shape (Figure 15).

Future works must dealt with the realization anc characterization of the proposed in-line coaxial-to-waveguide transition at Q-band. Given the thorough tolerance analysis, the expected range of variation of the most influential geometrical parameters can be controlled by using milling as manufacturing method. Alternative, more recent and appealing technologies, such as 3D printing [44] may not be able to satisfy the tight mechanical tolerances required at these high working frequencies. In particular, selective laser sintering can achieve a resolution of about 20-60 $\mu \mathrm{m}$ [45]. Furthermore the equipment can be costly.

\section{Conclusions}

In this work, an in-line coaxial-to-waveguide transition in Q-band has been proposed. The transition is $13.24 \mathrm{~mm}$ long, and the only increase in space occupation is due to a tapered backshort where a cavity is inserted. The transverse section size of the full waveguide is not exceeded. The modifications in the rectangular waveguide are reduced to the strict necessary to achieve the impedance matching with the coaxial cable and a suitable modal conversion. The critical design issues are studied through a tolerance analysis, reported in the result section.

The return loss keeps higher than $25 \mathrm{~dB}$ in the whole Q-band $(\mathrm{BW} \%=40.96)$, guaranteeing a good operation over the all required frequencies, and an acceptable margin to compensate the unavoidable manufacturing errors. The insertion loss (lower than $0.06 \mathrm{~dB}$ ) results sensibly better than in a typical microstrip-based in-line transition, due to the reduced dispersion in the coaxial end-launcher.

This improvement significantly facilitates the signal detection and processing, increasing the signal power level compared to the noise generated by the devices which constitute the receiving RF system. The results suggest the suitability of the proposed solution to develop an in-line transition with low losses by maintaining a very compact geometry and, in the same time, satisfying the requirements over a wide frequency band. This makes the proposed in-line coaxial-to-waveguide transition suitable for SFB antennas arrangement in radio-astronomy and satellite communication.

Author Contributions: Conceptualization: M.S., A.F. and G.M.; simulation: M.S.; validation: M.S., M.B.L.; analysis: M.S, A.F., T.P., and M.B.L.; data curation: M.B.L., T.P.; writing-original draft preparation: M.S.; writing—review and editing: M.S., M.B.L., A.F., T.P and G.M.; supervision: A.F. and G.M. All authors have read and agreed to the published version of the manuscript.

Funding: This research received no external funding. 
Institutional Review Board Statement: The work presented in this paper did not involve humans or animals.

Informed Consent Statement: Not Applicable.

Data Availability Statement: Data are available at: 10.6084/m9.figshare.14138042.

Acknowledgments: The authors are grateful to Stefano Fanti, for his precious help in improving the figures quality.

Conflicts of Interest: The authors declare no conflict of interest.

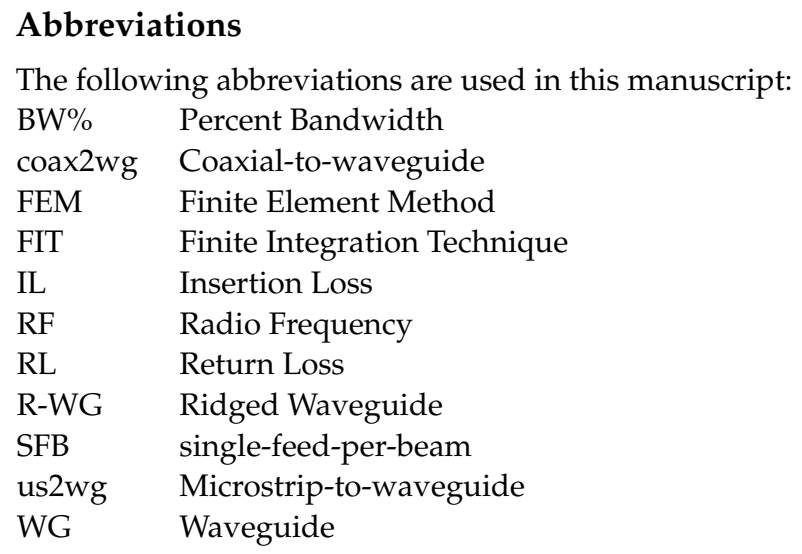

\section{Appendix A}

A further analysis of the device performance have been carried out by using another full-wave analysis simulation software, CST Microwave Studio, to compare the results provided by two different software which use different numerical methods to solve the electromagnetic problem and to provide another contribute to the result validation. Two different solvers have been tested in CST, the Time Domain Solver which is based on the Finite Integration Technique (FIT) to solve the Maxwell equations, and the Frequency Domain Solver which is based of the Finite Element Method (FEM). The simulation settings for both the tests are as follows in the Table A1.

Table A1. Simulation settings in CST Microwave Studio.

CST-MS - Time Domain Solver

Mesh Type: Hexaedral

Accuracy: $-50 \mathrm{~dB}$

Mesh properties

(a) 40 cell per wavelength

(b) Volume refinement: 6 additional cells around box

(c) Face refinement: minimum 6 cell across face

(d) Edge refinement: 6 additional cells around edges

CST-MS - Frequency Domain Solver Mesh Type: Tetrahedral

Mesh properties

(a) Adaptive Mesh refinement

(b) Maximum 15 passes

(c) S-parameter Threshold: 0.0025

The Figure A1 shows the Return Loss for the aforementioned CST simulations and the ANSYS simulation presented in the Result section (Figure 7), which has been tested with a Maximum Delta S equal to 0.001 and Discrete Sweep Type. The curves highlight a good matching between the three different simulations (all the curves keep higher than $24.25 \mathrm{~dB}$ ) and increases the confidence on the simulated results. 


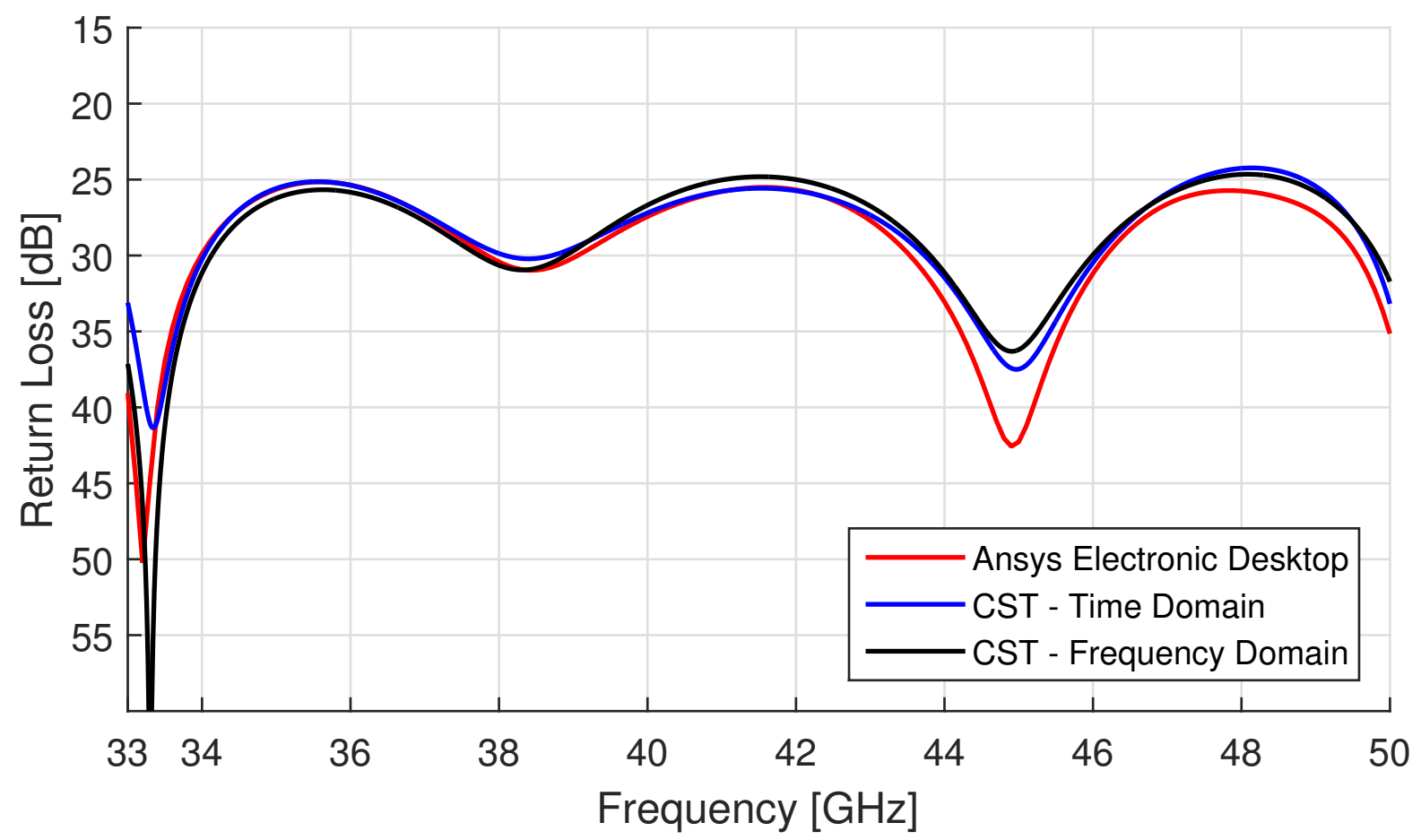

Figure A1. Comparison between the results provided by the different simulation software.

\section{References}

1. Wu, K.; Bozzi, M.; Fonseca, N.J.G. Substrate Integrated Transmission Lines: Review and Applications. IEEE J. Microwaves 2021, 1, 345-363. [CrossRef]

2. Balanis, C.A. Microstrip Antennas. In Antenna Theory: Analysis and Design, 3rd ed.; Balanis, C.A., Ed.; John Wiley \& Sons, Inc.: Hoboken, NJ, USA, 2005; pp. 811-862.

3. Pozar, D.M. Rectangular Waveguide In Microwave Engineering, 4th ed.; John Wiley \& Sons, Inc.: Hoboken, NJ, USA, 2012; pp. 110-121.

4. Helszajn, J.; Caplin, M. Impedance of ridge waveguide In Ridge Waveguides and Passive Microwave Components; Helszajn, J., Caplin, M., Eds.; The Institution of Engineering and Technology: London, UK, 2000; pp. 3-5.

5. Balanis, C.A. Horn Antennas. In Antenna Theory: Analysis and Design, 3rd ed.; Balanis, C.A., Ed.; John Wiley \& Sons, Inc.: Hoboken, NJ, USA, 2005; pp. 739-809

6. Li, Y.; Ge, L.; Wang, J.; Da S.; Cao, D.; Wang, J.; Liu, Y. 3-D printed high-gain wideband waveguide fed horn antenna arrays for millimeter-wave applications. IEEE Trans. Antennas Propag. 2019, 67, 2868-2877. [CrossRef]

7. Bolli, P.; Orfei, A.; Zanichelli, A.; Prestage, R.; Tingay, S.J.; Beltrán, M.; Burgay, M.A.R.T.A.; Contavalle, C.; Honma, M.; Kraus, A.; et al. An International Survey of Front-end Receivers and Observing Performance of Telescopes for Radio Astronomy. Publ. Astron. Soc. Pac. 2019, 131, 085002. [CrossRef]

8. Balanis, C.A. Circular Apertures. In Antenna Theory: Analysis and Design, 3rd ed., Balanis, C.A., Ed.; John Wiley \& Sons, Inc.: Hoboken, NJ, USA, 2005; pp. 683-689.

9. Balanis, C.A. Directivity. In Modern Antenna Handbook Balanis, C.A., Ed.; John Wiley \& Sons, Inc.: Hoboken, NJ, USA, 2008; pp. 16-21.

10. Montero, J.M.; Ocampo, A.M.; Fonseca, N.J.G. C-band multiple beam antennas for communication satellites. IEEE Trans. Antennas Propag. 2015, 63, 1263-1275. [CrossRef]

11. Buttazzoni, G.; Comisso, M.; Cuttin, A.; Fragiacomo, M.; Vescovo, R.; Gatti, R.V. Reconfigurable phased antenna array for extending cubesat operations to Ka-band: Design and feasibility. Acta Astronaut. 2017, 137, 114-121. [CrossRef]

12. Bhattacharyya A. K. and Goyette G. Smooth Wall Multimode Horns for High Aperture Efficiency-Theory, Design, and Applications. In Handbook of Reflector Antennas and Feed Systems-Volume 2; Rao S., Sharma S. K., Shafai L. Eds.; Artech House: Boston, MA, USA, 2013; pp. 117-119.

13. Lindgren, T.; Sautodehl, O.; Kilda, P.-S. Study of Cluster of Hard Horns Feeding an Offset Multi-beam Reflector Antenna for Dual Band Operation at 20/30 GHz. IEEE Antennas Propag. Soc. Symp. 2004, 3, 3015-3018. 
14. Bolli, P.; Orlati, A.; Stringhetti, L.; Orfei, A.; Righini, S.; Ambrosini, R.; Bartolini, M.A.; Bortolotti, C.L.; Buffa, F.; Buttu, M.; et al. Sardinia Radio Telescope: General description, technical commissioning and first light. J. Astron. Instrum. 2015, 4, 1550008. [CrossRef]

15. Orfei, A.; Carbonaro, L.; Cattani, A.; Cremonini, A.; Cresci, L.; Fiocchi, F.; Maccaferri, A.; Maccaferri, G.; Mariotti, S.; Monari, J.; et al. A Multi-Feed Receiver in the 18 to $26.5 \mathrm{GHz}$ Band for Radio Astronomy. IEEE Antennas Propag. Mag. 2010, 52,4, 62-72. [CrossRef]

16. Sorrentino, R.; Bianchi, G. Guide-to-coaxial cable transition. In Microwave and RF Engineering; Chang, K., Ed.; John Wiley \& Sons, Ltd.: Chichester, UK, 2010; pp. 198-203.

17. Pérez-Escudero, J.M.; Torres-García, A.E.; Gonzalo, R.; Ederra, I. A simplified design inline microstrip-to-waveguide transition. Electronics 2018, 7, 215. [CrossRef]

18. Zhang, Y.; Ruiz-Cruz, J.A.; Zaki, K.A.; Piloto, A.J. A waveguide to microstrip inline transition with very simple modular assembly. IEEE Microw. Wirel. Components Lett. 2010, 20, 480-482. [CrossRef]

19. Pérez-Escudero, J.M.; Torres-García, A.E.; Gonzalo, R.; Ederra, I. A Gap Waveguide-Based Compact Rectangular Waveguide to a Packaged Microstrip Inline Transition. Appl. Sci. 2020, 10, 4979. [CrossRef]

20. Wheeler, G. Broadband waveguide-to-coax transitions. In Proceedings of the 1958 IRE International Convention Record, New York, NY, USA, 21-25 March 1966; pp. 182-185.

21. Tang, R.; Wong, N.S. Multimode Phased Array Element for Wide Scan Angle Impedance Matching. Proc. IEEE 1968, 56 , 1951-1959. [CrossRef]

22. Deshp, M.D.; Das, N.S.; Sanyal, G.S. Analysis of an End Launcher for an X-Band Rectangular Waveguide. IEEE Trans. Microw. Theory Tech. $1979,27,731-735$. [CrossRef]

23. Saad, S.M. A more accurate analysis and design of coaxial-to-rectangular waveguide end launcher. IEEE Trans. Microw. Theory Tech. $1990,38,129-134$. [CrossRef]

24. Simeoni, M.; Coman, C.I.; Lager, I.E. Patch End-Launchers-A Family of Compact Colinear Coaxial-to-Rectangular Waveguide Transitions. IEEE Trans. Microw. Theory Tech. 2006 , 54, 2371-2380. [CrossRef]

25. Dix, J.C. Design of waveguide/coaxial transition for the band 2.5-4.1 Gc/s. Proc. Inst. Electr. Eng. 1963, 110, 253-255. [CrossRef]

26. Levy, R.; Hendrick, L.W. Analysis and synthesis of in-line coaxial-to-waveguide adapters. In Proceedings of the 2002 IEEE MTT-S International Microwave Symposium Digest, Seattle, WA, USA, 2-7 June 2002; Volume 2, pp. 809-811.

27. Boyns, J.E. Coaxial to Waveguide Transition for Horn Antenna. U.S. Patent No. 3,555,553, 12 January 1971. Available online: https:/ / patentimages.storage.googleapis.com/aa/9d/a2/8b526f29246e95/US3555553.pdf (accessed on 7 January 2021).

28. Tikhov, Y.; Song, I.S.; Won, J.H.; Kim, J.P. Compact broadband transition from double-ridge waveguide to coaxial line. Electron. Lett. 2003, 39, 530-532. [CrossRef]

29. Cano, J.L.; Mediavilla, A. Octave bandwidth in-line rectangular waveguide-to-coaxial transition using oversized mode conversion. Electron. Lett. $2017,53,1370-1371$. [CrossRef]

30. Carmel, N.; Elmakayes, D.; Matzner, H. Investigation of a Coax-to-Waveguide Transition Element. In Proceedings of the IEEE International Conference on Microwaves, Communications, Antennas and Electronic Systems COMCAS, Tel Aviv, Israel, 7-9 November 2011; Volume 27, pp. 1263-1275.

31. Hassan, E.; Norel, ; D.; Wadbro, E.; Berggren, M. Topology Optimisation of Wideband Coaxial-to-Waveguide Transitions. Sci. Rep. $2017,7,45110$. [CrossRef]

32. Nasr, M.A.; Kishk, A.A. Wideband Inline Coaxial to Ridge Waveguide Transition With Tuning Capability for Ridge Gap Waveguide. IEEE Trans. Microw. Theory Tech. $2018,66,2757-2766$. [CrossRef]

33. Simone, M.; Fanti, A.; Valente, G.; Montisci, G.; Ghiani, R.; Mazzarella, G. A Compact In-Line Waveguide-to-Microstrip Transition in the Q-Band for Radio Astronomy Applications. Electronics 2018, 7, 24. [CrossRef]

34. Kirschning, M.; Jansen, R.H. Accurate model for effective dielectric constant of microstrip with validity up to millimeter-wave frequencies. Electron. Lett. 1982 , 18, 272-273. [CrossRef]

35. Pozar, D.M. Summary of Transmission Lines and Waveguides. In Microwave Engineering, 4th ed.; John Wiley \& Sons, Inc.: Hoboken, NJ, USA, 2012; pp. 157-158.

36. Aljarosha, A.; Zaman, A.U.; Maaskant, R. A Wideband Contactless and Bondwire-Free MMIC to Waveguide Transition. IEEE Microw. Wirel. Components Lett. 2017, 27, 437-439. [CrossRef]

37. Collin, R.E. Rectangular Waveguide. In Foundations For Microwave Engineering, 2nd ed.; Dudley, G.D., Ed.; John Wiley \& Sons, Inc.: New York, NY, USA, 2000; pp. 181-182.

38. Pozar, D.M. Chebyshev Multisection Matching Transformers. In Microwave Engineering, 4th ed.; John Wiley \& Sons, Inc.:Hoboken, NJ, USA, 2012; pp. 256-261.

39. Helszajn, J.; Caplin, M. Impedance and propagation in ridge waveguides using the transverse resonance method. In Ridge Waveguides and Passive Microwave Components; Helszajn, J., Caplin, M., Eds.; The Institution of Engineering and Technology: London, UK, 2000; pp. 26-46.

40. Collin R.E. Quarter-Wave Transformers. In Foundations For Microwave Engineering, 2nd ed.; Dudley, G.D., Ed.; John Wiley \& Sons, Inc.: New York, NY, USA, 2000; pp. 343-346.

41. Robinson, J.; Rahmat-Samii, Y. Particle swarm optimization in electromagnetics. IEEE Trans. Antennas Propag. 2004, 52, 397-407. [CrossRef] 
42. Simone, M.; Fanti, A.; Mazzarella, G. Ridge waveguide optimization with PSO algorithm. J. Electromagn. Waves Appl. 2015 , 29, 199-209. [CrossRef]

43. Zhang, B.; Zirath, H. Metallic 3-D Printed Rectangular Waveguides for Millimeter-Wave Applications. IEEE Trans. Components Packag. Manuf. Technol. 2016, 6, 796-804. [CrossRef]

44. García-Martínez, H.; Ávila-Navarro, E.; Torregrosa-Penalva, G.; Rodríguez-Martínez, A.; Blanco-Angulo, C.; de la Casa-Lillo, M. Low-Cost Additive Manufacturing Techniques Applied to the Design of Planar Microwave Circuits by Fused Deposition Modeling. Polymers 2020, 12, 1946. [CrossRef]

45. Addamo, G.; Peverini, O.A.; Manfredi, D.; Calignano, F., Paonessa, F.; Virone, G.; Tascone, R.; Dassano, G. Additive Manufacturing of Ka-Band Dual-Polarization Waveguide Components. IEEE Trans. Microw. Theory Tech. 2018, 66, 3589-3596. [CrossRef] 